
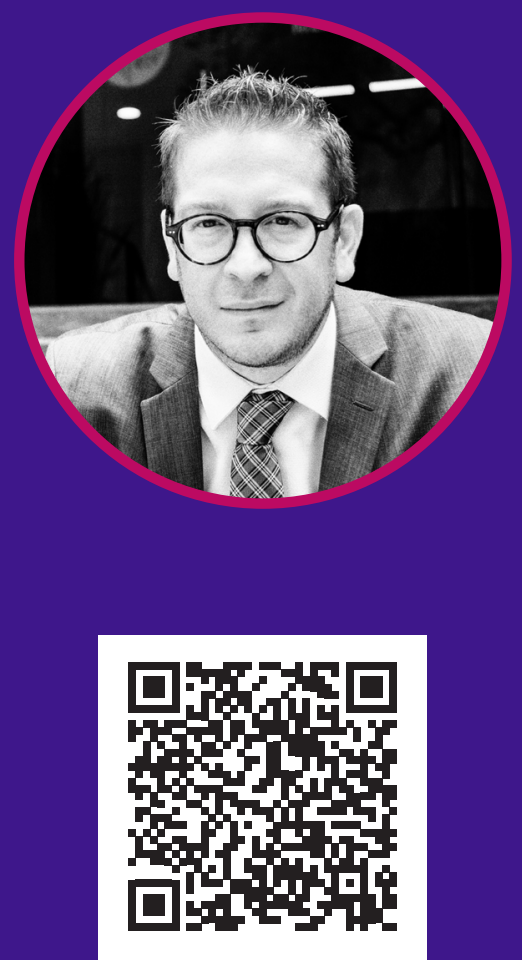

Videopresentación

\section{Stranger Things y la expansión del relato como práctica transmedia}

\author{
Stranger Things and the expansion of stories as transmedia \\ practice
}

\section{Mtro. Bernardo Flores Heymann}

Universidad Panamericana

bfloresh@up.edu.mx

Recibido: 15 de agosto de 2020.

Received: August 15th 2020.

Aceptado: 19 de septiembre de 2020.

Accepted: September 19th, 2020.

(c) $९ €$ Esta obra está bajo una licencia internacional Creative
Commons Atribución-NoComercial-SinDerivadas 4.0.

DOI: https://doi.org/10.21555/rpc.voi2.2340

Cómo citar: Flores Heymann, B. . (2020). Stranger Things y la expansión del relato como práctica transmedia. RPC, (2), 96-105. https://doi.org/10.21555/rpc.v0i2.2340

Revista Panamericana de Comunicación, Año 2, N. 2, julio-diciembre 2020, pp. 96-105.

\section{RESUMEN}

Stranger Things es una serie de televisión web que ha expandido su narrativa a través de diversos medios y formatos. El objetivo de este artículo es analizar la expansión del relato desde la perspectiva de los siete principios de las narrativas transmedia creado por Henry Jenkins, por medio de la documentación e indagación de dichas ramificaciones.

Palabras-clave: Stranger Things, narrativas transmedia, expansión del relato, integración mediática, Henry Jenkins.

\section{A B STRACT}

Stranger Things is a web television series that has expanded its narrative through various media and formats. The aim of this article is to analyze the expansion of the story from the perspective of Henry Jenkins' seven principles of transmedia storytelling, through the documentation and study of these ramifications.

Palabras-clave: Stranger Things, transmedia storytelling, Spreadability, media integration, Henry Jenkins. 
M arshall McLuhan vaticinó que llegaría un punto en el la tecnología estaría tan a la mano de los consumidores que estos mismos se convertirían en productores de contenidos (1972). Esto sentó la base para que en 2003, un profesor del Massachusetts Institute of Technology (MIT), publicara "Narrativas Transmedia" (Transmedia storytelling) un ensayo con en el abría la puerta al estudio y análisis de las nuevas experiencias de entretenimiento que, aunque en ciernes, comenzaban a moldear el mundo. En este texto se hace una de las primeras menciones sobre la relevancia que la participación de la audiencia tiene en el desarrollo de historias y narrativas para el siglo XXI.

En 2006 el mismo profesor, el Dr. Henry Jenkins, presentó la base que sustenta la producción informativa y de entretenimiento en nuestro tiempo: La Cultura de la Convergencia. Para 2009, una vez desarrollada la base que permite la comprensión fenomenológica del consumo de medios y nuevos medios gracias a internet, el mismo Jenkins dio a conocer los Siete Principios que moldean cualquier experiencia incrustada en una narrativa transmedia (2009).

El primer objetivo de este artículo es el analizar la fragmentación narrativa que la producción de Stranger Things, una serie televisiva creada por Netflix, desarrolló en en diferentes medios y formatos. Para cumplir con este objetivo se documentarán las expansiones desarrolladas específicamente para México, pero se cruzarán con información disponible que la misma producción, o bien Netflix, ha desarrollado en otros territorios con el propósito de diseminar su narrativa en diveros nichos.

Una segunda meta consiste en esclarecer si estas fragmentaciones cumplen con el primer principio de las narrativas transmedia: Expansión Vs Profundidad.

Para cumplir con dicho propósito, se tomarán como base teórica las definiciones de narrativa transmedia postuladas por diversos autores, pero tomando como referencia a Jenkins como pilar del concepto terminológico. Asimismo, considerarán los postulados de la cultura de la convergencia para enmarcar la documentación de los relatos analizados alrededor de Stranger Things.

\section{NETFLIX, LA RUPTURA DE PARADIGMAS Y PREPONDERANCIA EN EL STREAMING}

A nivel mundial Netflix es la plataforma de streaming de video dominante con más de 182 millones de suscriptores. Prime Video, de Amazon, se posiciona en segundo lugar con más de 150 millones, seguido por HBO con aproximadamente 140 millones de usuarios. En México la historia es similar con datos que calculan desde desde los 6 y hasta los 18 millones de cuentas para Netflix, según la medición que alguna firma haga. Claro Video, de América Móvil, se posiciona en segundo lugar y Prime Video en tercero.

Según datos publicados por diversos medios de comunicación (El Sol de México, 2019; Excélsior, 2018; Forbes, 2020), México es el segundo país con más suscriptores de Netflix, y el quinto que más horas de contenido consume, aunque los datos exactos entre las publicaciones varían, la jerarquía se mantiene.

Así, en 2018, en México la plataforma OTT sumaba 6.6 millones de usuarios según datos revelados por el INEGI, pero acorde a la consultora Kantar hasta julio de 2019 el número estaba más bien cerca de los 18 millones de cuentas.

Este cambio en el consumo de contenidos, de ver televisión abierta o de cable, con servicios de streaming, tiene que ver con la naturaleza cambiante de los usuarios buscando nuevas experiencias de entretenimiento en un mundo de convergencia mediática (Jenkins, 2006).

Uno de los principales precursores de estos cambios ha sido Netflix que, a partir de 2007 permitió una nueva experiencia televisiva al permitir los maratones de series (binge viewing) y luego, cuando en 2013 rompió el formato tradicional de decisiones de producción con el lanzamiento de House Of Cards, una de las series más aclamadas de la plataforma, pero para la cual la decisión de producirla estuvo sustentada completamente en un algoritmo (Smith \& Telang, 2016).

Netflix ha producido películas, series, documentales y hasta podcasts por medio de la interpretación de los datos que recopilan de sus usuarios en todas las plataformas en las que están presentes, desde redes sociales, aplicaciones móviles y sitios de internet. 
Si bien se trata de series y filmes que siguen un proceso de producción tradicional, la experiencia de creación es completamente novedosa para el usuario, así como el resultado al abordar temas nuevos, tabúes, o socialmente alarmantes (Almaguer, 2019).

Una de las producciones más trascendentes de Netflix -hablando específicamente de su consumoha sido Stranger Things que, según datos publicados por la propia plataforma, el estreno de la tercera temporada rompió récords de audiencia: más de 40 millones de personas empezaron la tercera temporada durante los cuatro días siguiente a su estreno y 18.2 millones de usuarios terminaron de ver los ocho episodios en el mismo lapso.

Netflix \$ @etflix.8 jul. 2019

@Stranger_Things 3 is breaking Netflix records!

40.7 million household accounts have been watching the show since its July 4 global launch - more than any other film or series in its first four days. And 18.2 million have already finished the entire season.

Q $3,9 \mathrm{mil}$ 七】 $38,7 \mathrm{mil}$ $207,5 \mathrm{~m}$ $\uparrow$

Fig. 1 Captura de pantalla del 13 de agosto de 2020 del Tweet de Netflix hablando del éxito de la tercera temporada de Stranger Things. El link al mismo está disponible en las referencias.

\section{LOS ORÍGENES DE STRANGER THINGS}

Stranger Things es una producción para televisión web (Lobato, 2019) coproducida y distribuida por Netflix que se estrenó en 2016. Esta serie se desarrolla en la década de 1980, en el pueblo ficticio de Hawkins, Indiana, Estados Unidos, luego que Will Byers, un niño de 12 años, desaparece en circunstancias muy extrañas.

La televisión web se puede definir como contenido episódico original producido exclusivamente para su transmisión usando alguna interfaz conectada a la World Wide Web (William, 1994).

Aunque el principal giro de Netflix es la distribución de contenidos audiovisuales por medio de su plataforma de video bajo demanda, desde 2011 tomó la decisión de generar contenidos propios, idea que se materializó en 2013 con el lanzamiento de House of Cards, seguida de Orange is the New Black. Estas dos series reiteraron en la compañía el interés de sus usuarios por contenidos nuevos, disruptivos y creados a partir del estudio de algoritmos. Además, con el lanzamiento de más producciones propias, retaron diversos dogmas de la industria cinematográfica y televisiva del mundo. Para el análisis de tal caso existe la literatura disponible que menciona cómo la industria televisiva y de Hollywood intentó impedir la participación de las producciones cinematográficas en los certámenes más relevantes del mundo (Selter, 2013).

En 2015, la compañía de distribución apostó por la historia de los hermanos Matt y Ross Duffer y sentó el precedente de abrir sus puertas a nuevos talentos en cuestión de guionismo, producción y dirección. Así pues, compró los derechos de la serie Stranger Things, originalmente titulada Montauk. En julio de 2016 se lanzó la primera temporada que, desde la semana de estreno, recibió buenas críticas, lo que le permitió renovarse para una segunda temporada que llegó a las pantallas en 2017.

Las primeras dos temporadas de Stranger Things se desarrollan entre 1983 y 1984 cuando un niño desaparece de manera misteriosa en un pueblo de Indiana, Estados Unidos, en el que el gobierno de dicho país tiene un laboratorio. Una chica, llamada Once (Eleven o El, en inglés) y también conocida como Ce (en español), que posee poderes psíquicos, como la bioquénesis y clarividencia, aparece para ayudar a los amigos de Will a encontrarlo.

La tercera temporada, lanzada en el verano de 2019, sucede en el mismo pueblo ficticio de Indiana, Hawkins, pero ahora los protagonistas están entrando en la adolescencia y tendrán que trabajar en equipo para evitar que El Mundo del Revés (Upside Down), de donde provienen todas sus amenazas, los separe y destruya. Aunque se confirmó una cuarta temporada, debido a la pandemia global de Covid19, la producción se detuvo.

Narrativa, ética y estética de la serialidad televisiva 


\section{LA CONVERGENCIA, LAS NARRATIVAS TRANSMEDIA Y SUS PRINCIPIOS}

Para Jenkins -y en general para los usuariosun solo medio ya no satisface las necesidades de su estilo de vida (2003) y por tanto las audiencias están en constante búsqueda de nuevas experiencias de entretenimiento (2006).

Para poder comprender el desarrollo de narrativas y franquicias transmedia es necesario ahondar someramente en las características de la cultura de la convergencia: la inteligencia colectiva entendida como el conocimiento común aglutinado para generar nuevo conocimiento, y por tanto la participación de los usuarios en el desarrollo de las historias por medio de la producción de nuevos contenidos alrededor de las ideas originales. La convergencia mediática, que se definirá mejor en el siguiente punto, pero comprendida como la fusión de diversos jugadores y sus respectivos formatos con la finalidad de mejorar la experiencia del usuario con contenidos novedosos, expansivos e interesantes. Finalmente, la cultura convergencia en sí misma: la facilidad de conectarse inmediatamente a todo, la hipertextualidad y la combinación de formatos para crear nuevos contenidos (Jenkins, 2006).

Tomando estas tres características como cimiento Jenkins crea siete principios que cualquier narrativa debiera considerar, aunque para el objeto de este artículo, se tomará en cuenta el primero: Expansión Vs. Profundidad.

Los otros seis principios son Continuidad Vs. Multiplicidad comprendidos superficialmente como la coherencia y la posibilidad de ingresar a narrativas alternativas de la misma narrativa. El tercero, que se incluirá brevemente en este análisis, es el de Inmersión Vs. Extracción, que es la facilidad que la producción ofrece de vivir una parte del mundo ficticio al tiempo que se saca algo para ingresarlo al mundo real. La Construcción de mundos está ligado al principio previo y comprende la facilidad con la que se puede crear o desarrollar una experiencia. La Serialidad consiste en que cada narrativa, aunque independiente, tiene un orden en su arco narrativo. Por su parte la Subjetividad radica en la posibilidad de entender, ver y explorar la narrati- va original, o cualquier fragmentación, desde perspectivas alternativas, ya sea de personajes o historias. El último principio, la Ejecución, tiene diversas acepciones -muy discutibles todas que se pueden analizar en futuros trabajos- pero está definida como la posibilidad de integrar las historias alternativas desarrolladas por los usuarios al universo narrativo original.

\section{EL SALTO DEL FORMATO TELEVISIVO, A LOS DISPOSITIVOS MÓVILES, A LAS CONSOLAS DE VIDEOJUEGOS: LA EXPANSIÓN.}

Parte de la convergencia antes mencionada incluye la colaboración de las industrias y compañías productoras, pero más que cooperación emulan alianzas que permitan la supervivencia ante Netflix que domina el metamedium (Scolari, 2004) en cuanto a distribución y producción de contenido audiovisual, cinematográfico y televisivo. Las grandes televisoras y casas productoras, como Caracol, en Colombia; Argos, en México; o NBC, en Estados Unidos, además de producir contenidos para sus propiedades, están contratadas por Netflix para crear contenidos originales en una lucha por su supervivencia.

Quizá el primer caso registrado de expansión de relato de la televisión a los dispositivos móviles fue con la serie 24, protagonizada por Kiefer Sutherland. En 2005 los creadores de la serie experimentaron con la creación de mobisodes -episodios de la serie, desarrollados para dispositivos móviles, con una duración muy corta (un minuto).

Conspiracy, nombre del spin-off(subproducto), creó 24 episodios, de un minuto, que cuentan una historia alternativa, pero relacionada con la temporada cuatro de la serie. Sin embargo, la tecnología del momento, tanto en los dispositivos, como para el streaming, y la creciente industria de la piratería, hicieron que se cancelara el proyecto.

Stranger Things comenzó como un producto de televisión web pero para su segunda temporada, lanzada en 2017, expandió el relato, y con esto la experiencia del usuario a través de Stranger Things: The Game, un juego de video disponible y gratuito para dispositivos móviles con sistema operativo Android o iOS. El RPG 
(role playing game) creado a partir de la serie mantenía la ambientación de los 80 , con gráficos en ocho bits, y permitía realizar 30 diferentes misiones con hasta siete personajes. Aunque mantenía la dinámica y narrativa de la historia, los jugadores podían indagar y explorar detalles no ofrecidos por la serie televisiva.

La razón detrás de la creación del juego no está clara, sin embargo, existen fuertes evidencias para considerar que la estrategia de comunicación de la serie incluía mantener a los usuarios entretenidos y conectados con la narrativa durante los largos periodos de producción. En 2017 el mismo juego se lanzó para Play Station 4 la versión de realidad virtual, Stranger Things: The VR Experience.

La expansión narrativa, el brinco de un medio a otro, es solamente natural bajo las circunstancias que la comunicaciónn digital permitió (Negroponte, 1995) y este cambio paradigmático de consumo está sustentado con cambio del modelo mediático tradicional en el que se transmite de uno a muchos, al modelo actual que va de muchos prosumidores a muchos otros ( $\mathrm{SCO}$ lari, 2008).

La posibilidad de que un desarrollo narrativo atraviese -cruce- y combine los viejos lenguajes y medios, con los nuevos es solo permitido en mundo de nodos, interconectado, remediado y convergente (Orihuela, 2003. Jenkins, 2006. Scolari, 2008.). Pero más allá de la teoría, la práctica implica una reconfiguración en la estructura de producción de cualquier narrativa considerando mantener cautivas a las audiencias en todas las plataformas posibles con un bajo mismo título.

Con la llegada de la tercera temporada llegó también Stranger Things 3: The Game para teléfonos inteligentes y dispositivos móviles mostrando una nueva versión del juego, amplificada en cuanto a opciones, personajes y aventura. Para esta entrega el videojuego ya costaba casi seis euros incrementando el valor económico de la franquicia. Aunado a esto, Netflix participó en el E3 2019 (Electronic Entertainment Expo), en Los Ángeles, donde anunció además cambios en las versiones de su juego para Xbox, PS4, PC, Mac y Nintendo Switch.

\section{LA FRAGMENTACIÓN PUBLICITARIA: LA NARRATIVA DE JAIME MAUSSAN}

La posibilidad de expandir el relato es uno de los siete principios que cualquier narrativa enfocada en entretenimiento debe cumplir para que se pueda llamar transmedia según Henri Jenkins (2009). El principio contrapuesto de Expansión contra Profundidad (Spreadability Vs Drillability) "se refiere a la capacidad del público para participar activamente en la circulación del contenido de los medios a través de las redes sociales y en el proceso expandir su valor económico y cultural" (Jenkins, 2009). Esto se traduce en que la expansión del relato está en manos del público y, por tanto, entre más contenidos diseminados, pero integrados en una narrativa, el valor de la marca o franquicia se potenciará. Los fans hablaron mucho en redes, y no sólo de la serie, también de los videojuegos, pero hubo una nueva extensión del relato promovida por la parte publicitaria.

Desde el lanzamiento de Stranger Things en México, en 2016, la producción de la serie tropicalizó la publicidad en dos sentidos: hacerla más atractiva para los usuarios locales relacionando su producto con algo del país o región y, en segundo término, con la finalidad de apelar a la nostalgia y sus recuerdos de la década de 1980.

La fusión de estos dos elementos terminó con la contratación del ufólogo e investigador de eventos paranormales Jaime Maussan para sus anuncios en México. Maussan es un periodista mexicano que fue corresponsal en Estados Unidos para el programa de Domingo a Domingo y 24 Horas. Sus investigaciones y reportajes sobre el cambio climático, medio ambiente y la naturaleza fueron muy relevantes durante los 80 y en la actualidad sigue siendo una voz relevante en esos temas. Sin embargo, se le conoce más por sus investigaciones relacionadas con hechos paranormales y el fenómeno de objetos voladores no identificados (Ovnis) que, por varias décadas fueron razón de señalamientos a su trabajo, hasta que el Pentágono de Estados Unidos desclasificó tres vides de ovnis en 2020.

En la primera aparición y colaboración entre Netflix y Maussan, el periodista se encuentra en Haw- 
kins, el pueblo ficticio, investigando la desaparición del niño Byers. Si bien esto entra directamente en el ámbito publicitario y tiene el sello de la firma de Los Gatos, California, la interpretación subsiguiente es que con este acercamiento se tiene clara la intención de expandir el relato de forma local.

De hecho, los productores de la serie han apelado a temas y programas icónicos de los 80 en los distintos países que promueven la serie, algunos con éxito como lo fue en México (más de 300 mil reproducciones al 14 de agosto de 2020 del primer promocional de 2016), y otros sin tanta aceptación, como en España.

Este primer intento de expansión del relato televisivo tuvo buena recepción entre la audiencia y para el estreno de la segunda temporada, en 2017, Maussan participó nuevamente, ahora buscando al Chupacabras e intentando proteger a tres de los protagonistas de la serie. De hecho, el tratamiento que se le ha dado a los esfuerzos de mercadotecnia tiene un formato de miniserie, ya que, para cada una de las temporadas, el anuncio se maneja como un episodio más, que complementa la narrativa general, pero tiene una historia alternativa a la narrativa original, en este caso Stranger Things.

Para la tercera y última temporada, disponible al momento de la entrega de este texto, el periodista participó de nueva cuenta en la campaña publicitaria junto con Cositas, un personaje de la televisión mexicana que presentaba manualidades en diferentes programas y espacios dirigidos a una audiencia joven. En el video se puede apreciar la misma producción ambientada en los 80 y que da entrada a la nueva temporada.

Alma Gómez Fuentes, la persona que da vida al personaje de Cositas, tuvo participación en diversos canales y programas de televisión desde 1988 hasta 2007. Durante algún tiempo de su vida de dedicó a hacer presentaciones privadas para despedidas de solteras, baby showers, entre otros.

La cultura de la convergencia exige la colaboración entre los entes mediáticos por sobrevivir (Jenkins, 2006) y es así como a partir de una breve aparición de poco más de cuatro minutos, Cositas regresó a los nuevos medios (Marshall, 2004) con un canal de YouTube en el que continúa enseñando manualidades y en TikTok, siguiendo las reglas digitales de creación y difusión de los nuevos medios, pero mezclados con el lenguaje de los llamados medios viejos, o tradicionales.

Retomando los principios de Jenkins sobre las narrativas transmedia, en un artículo titulado Transmedia Education: the 7 Principles Revisited, Jenkins propone una adecuación a su definición previa de expansión: "se refiere a un proceso de dispersión: escanear a través del panorama de los medios en busca de bits de datos significativos" (Jenkins, 2010). Y al final lo establece como la capacidad y el grado en que el contenido se puede compartir y los factores que motivan a una persona a compartir ese contenido dejando claro que es necesario motivar a los usuarios a diseminar de manera activa todas y cada una de las producciones.

\section{LA INMERSIÓN A HAWKINS, INDIANA COMO ELEMENTO PARA LA EXPANSIÓN DEL RELATO}

Siguiendo la retórica de los principios de Jenkins se cuenta también con la necesidad de la inmersión a la narrativa con la finalidad de que los espectadores y usuarios vivan ese mundo ficticio 0 alternativo en la realidad. Hay diversos ejemplos de inmersión: desde la visita a los parques de Disney en los que se puede tener una experiencia vívida de alguna de sus producciones, como Star Wars hasta convivir con alguna princesa, incluyendo en este ámbito también las experiencias desarrolladas en España para el lanzamiento de las diferentes temporadas de Juego de Tronos (Pratten, 2015).

"En la inmersión, el consumidor entra en el mundo de la historia (como los parques temáticos)", dice el texto revisado sobre sus principios originales. Stranger Things no sólo expandió el relato, pero también logró en julio de 2019 una experiencia inmersiva al lado de otra marca que patrocinó la experiencia. La activación publicitaria consistía en visitar, en grupos de tres y hasta 10 personas, 10 cuartos icónicos de la serie. La dinámica, más allá de ser una visita multimedia, permitía sumergirse de lleno en la historia por lo que los usuarios debían resolver diversos acertijos.

Stranger Rooms, nombre que recibió la activación en la Ciudad de México, utilizó el esquema de los escape rooms en la que los participantes deben trabajar en equipo para encontrar las respuestas que les permi- 
tan salir de la habitación en la que están antes de que algo les suceda. La atracción estuvo abierta del 5 al 21 de julio y ofrecía a los asistentes la oportunidad de vivir dentro de las habitaciones de los personajes de la serie. Con narrativas expandidas, cada equipo tenía 15 minutos para resolver un enigma o adivinanza dentro de uno de los cuartos más representativos, como el sótano de Mike, antes de que un monstruo -el Demogorgon (Desuellamentes, en castellano)- "se los comiera". La experiencia por supuesto no terminaba con la muerte del asistente, ya que los participantes podían tomarse fotografía dentro de los cuartos y quedarse ver alguno de los episodios de la serie.

\section{LA ADAPTACIÓN DEL RELATO Y EL RETO DE LA PROFUNDIZACIÓN EN LA NARRATIVA}

Uno de los argumentos discutibles de los principios establecidos por Jenkins es la participación de los usuarios en la expansión de la narrativa. Por un lado, están los que argumentan que la participación debe ser guiada (Pratten, 2011) y otros autores, como Scolari, postulan que esto depende de la estructura que se establezca (2013). Sin embargo, aunque la participación de la audiencia se puede medir a partir de rendimiento (Performance) en más bien claro que es necesario entenderla desde la perspectiva de la profundidad (Drillability), el complemento opuesto de la expansión.

La temporada tres de Stranger Things, además de romper récords internacionales de audiencia (según datos no públicos de Netflix), crear experiencias inmersivas y ampliar los relatos a nuevas plataformas, como lo hicieron con los videojuegos, en México optaron por lanzar un podcast exclusivo de la serie: La Radio Extraña.

Habiendo definido la expansión como proceso de diseminación, la profundidad está por tanto enfocada "a la habilidad de una persona para explorar a profundidad el pozo de extensiones narrativas cuando se enfrentan con alguna ficción que capture en realidad sus sentidos" (Jenkins, 2010).

Una constante que se mantiene en las producciones es que, cuando se desea expandir el relato muchas veces se termina con una adaptación de este. En este sentido, la adaptación narrativa cae en el término crossmedia, como es el caso de las adaptaciones de Harry Potter de los libros al cine, y no en el de transmedia.

Con el estreno de la tercera entrega de la serie de misterio, W RADIO, una estación radiofónica de la ciudad de México anunció: "Ahora Stranger Things llega a la radio como en los años 80 cuando las series eran escuchadas en antena. La primera serie de Netflix adaptada a este formato y podrás escucharla el 1, 2 y 3 de julio a las 8:00 PM por W RADIO en el 96.9 FM o 900 AM Ciudad de México".

Los tres episodios que se realizaron fueron una adaptación de cada una de las temporadas a un formato de radionovela muy corta sin ofrecer detalles extras o narrativas expandidas. El episodio uno tuvo una duración de 7:23 minutos, el segundo de 6:51 y el tercero de 8:35. No hay detalles o datos para evaluar el éxito y fracaso de la producción, pero dada la limitación para escucharlo fuera de la transmisión en vivo, puede inferirse que se trató únicamente de una prueba expansiva.

Algunos sitios informativos dieron a conocer durante julio de 2019 que Netflix había lanzado un nuevo podcast, en inglés, en el que ahondaría en detalles, dudas y temas que los fans tenían y que estaría disponible en Spotify y Apple Podcast. El podcast ya no se encuentra disponible en la plataforma de Apple y el url fue sustituida por un programa similar al descrito previamente, pero que ahonda en los detalles de The Umbrella Academy (Netflix, 2019).

Behind the Scenes: Stranger Things 3 / The Starcount Mall el podcast que se lanzó con la tercera temporada está disponible en el canal de YouTube de Netflix.

El podcast en cuestión es una mezcla de entrevistas con los actores, productores, guionistas, entre otros que incluye comentarios sobre lo que sucedió en la producción de la tercera temporada de la serie y está conducido por Dan Taberski, un periodista estadounidense conocido por desarrollar podcasts a partir del resultado de sus investigaciones periodísticas.

El podcast, someramente, permite profundizar en la vida de los personajes, pero en realidad es más bien un complemento a la producción que no aporta algo a la narrativa por lo que la expansión del relato se mantiene limitada. Parte de la comunicación relacionada con esta producción sonora rezaba que "si estás en 
busca de contenido sobre tu serie favorita para llenar el vacío de tu corazón, este podcast es para ti” (Núñez, A, 2019).

El lugar en el que sí es posible indagar más sobre los acontecimientos y vida de Hawkins, Indiana, es en los más de 18 podcasts registrados en Spotify creados por seguidores de la serie. Asimismo, es posible profundizar en las más 12 mil páginas y foros de debate (según los resultados de Google al buscar Stranger Things Fandom, Grupos de debate, fans Stranger, Things, etcétera) que en los mismos esfuerzos expansivos que ha hecho la franquicia.

Es posible por tanto decir que, aunque el relato se expanda, es necesario siempre contar con suficientes refuerzos que permitan desarrollar y crear contenidos suficientes para que los usuarios puedan indagar más sobre la ficción, sus personajes, o bien, la historia.

\section{OTRAS EXPANSIONES NARRATIVAS DE STRANGER THINGS}

A partir del modelo creado por Netflix, se han creado nuevos modelos que se autonombran el "Netflix de algo más". Por ejemplo, Yaconic o Bookmate son relacionados como el Netflix de los libros; DAZN es Ilamada el Netflix de los deportes, y así de manera consecuente. Existe ya esa acepción para videojuegos, periódicos, conciertos, y muchos más. Lo que no existe aún es un Netflix para los podcasts. Pero Netflix aparentemente va por ellos.

Netflix empezó a generar podcasts como los detrás de cámaras antes mencionados, así como los esfuerzos publicitarios del tipo La Radio Extraña (W Radio, 2019), que acompañan los lanzamientos de sus series y temporadas. Pero también el formato de radionovela, o serie radiofónica está ya dentro de las producciones y, junto con Spotify lanzaron The Only Podcast Left (EI último podcast que queda). Esta producción sí es complementaria e independiente a la serie posapocalítpica Daybreak (Netflix, 2019), que introduce nuevos personajes y versa sobre unos adolescentes que intentan publicar un podcast para hablar de la situación que están viviendo. Este tipo de esfuerzos aún no se tienen en español, pero se ha especulado sobre la posibilidad de hacerlo con Élite, o La Casa de Papel, las dos series más exitosas en castellano producidas por Netflix.

En mayo de 2019, la empresa productora y distribuidora de California, Netflix, lanzó Nada que ver, un programa en el que "se harán recomendaciones de lo que no te puedes perder, estrenos, joyas y una que otra rareza", como un intento más de ofrecer detalles profundos a las expansiones de los relatos.

Si bien Nada que ver no cuenta con sobrados "pozos de extensiones narrativas" para los usuarios, es un esfuerzo interesante para impulsar la expansión, subjetividad y la participación constante de las audiencias.

\section{CONCLUSIONES}

La indagación de las fragmentaciones narrativas de Stranger Things reveló que, además de la serie de televisión web creada por Netflix, también incluyó diversas producciones alternativas que ayudaron a expandir el relato. Se encontraron para justificar este punto diversos juegos de video para dispositivos móviles, además de consolas; también se encontró un ejercicio publicitario que contaba una historia relacionada, pero alternativa que se dio conocer por YouTube. Aunado a esto, la producción diseñó una experiencia física que tomaba la narrativa original para crear un mundo simultáneo. El último hallazgo consistió en un par de producciones sonoras en formato podcast que, si bien no funcionaron como fragmentos independientes que incrementaran el relato narrativo, sí impulsaron el alcance en plataformas alternativas.

Durante la investigación se observó que la ejecución práctica muestra que cada vez son más las producciones audiovisuales, televisivas y cinematográficas que encuentran valor de alcance y económico al producir expansiones para mantener a las audiencias cautivas dentro de sus narrativas. Por tanto, no es aventurado pensar que por la galaxia terminológica que se ha dado al concepto de transmedia (Mora, 2014), el futuro de esta dependa de la articulación moderna de términos antiguos como multimedialidad, digitalización e hipertexto (Scolari, 2019) y una integración mediática termine por definir la transmediación, sus narrativas y efectos socioculturales. 
En cuanto al segundo objetivo, es claro que las fracturas narrativas sí cumplen con el principio de la Expansión Vs. Profundidad, sin embargo existen áreas de oportunidad. En cuanto al primer elemento del principio, la expansión, en todas las experiencias narrativas identificadas se cumple el propósito de diseminar la narrativa y brindar más valor económico y cultural al mundo desarrollado, así como la capacidad del público para participar en la circulación del contenido en diversos medios. Sin embargo, la parte de la profundidad, definida como "la habilidad de una persona para explorar a profundidad el pozo de extensiones narrativas cuando se enfrentan con alguna ficción que capture en realidad sus sentidos", no se complementa de forma definitiva. Las expansiones creadas por W RADIO, así como por Netflix, en Estados Unidos, no permiten profundizar suficiente en el mundo ficticio de Stranger Things.
Una posibilidad para ahondar más en el tema consiste en las dificultades específicas para crear narrativas más profundas que permitan facilitar la expansión narrativa. Asimismo, otro espacio en el que se podría investigar sería para conocer los esfuerzos de Netflix, o de otras plataformas de streaming, como HBO y su Juego de Tronos, para crear franquicias transmedia específicas, adecuadas y comprender el tamaño de la participación que la audiencia tiene en ellas. I

\section{REFERENCIAS}

» Almaguer, M. (2019, 12 noviembre). Netflix tiene un objetivo: hablar sobre temas tabú. Glamour. https://www.glamour. es/placeres/cultura/articulos/netflix-impacto-positivo-temas-tabu-por-13-razones-to-the-bone-atypical/27203

» Asociación de Internet MX. (2019, July). 15 Estudio sobre los Hábitos de los Usuarios de Internet en México 2018 (No. 15). https:// www.asociaciondeinternet.mx/

» Bernardo, R. R. (2015, 16 marzo). Los 7 principios de la narrativa transmedia según Henry Jenkins. Transmedia Experience. http:// transmediaexperience.com/es/2015/03/los-7-principios-de-la-narrativa-transmedia-segun-henry-jenkins/

» Bottini, P. (2019, 3 mayo). Los 7 principios de Henry Jenkins - Patricia Bottini. Medium. https://medium.com/@patriciaesculpi/los7-principios-de-henry-jenkins-39f3113a82ba

» Conducttr. (s. f.). Game of Thrones - Conducttr. Transmedia Storyteller Ltd. https://www.conducttr.com/success-stories/game-of-thrones/

» Coppel, E. (2017, 17 noviembre). Jaime Maussan y los niños de "Stranger Things» buscan al chupacabras en este video. Verne. https://verne.elpais.com/verne/2017/11/17/mexico/1510943309_878828.html
» Corona, J. (2016). ¿Cuándo es transmedia?. Icono 14, volumen 14, pp. $30-48$.

» Del Barco, L. (2020, 4 septiembre). Netflix estrenará esta semana su primer podcast de ficción.

» Hipertextual. https://hipertextual.com/2019/11/netflix-podcast-ficcion-daybreak

»Estavillo, M. E. (n.d.). Los Servicios OTT: provisión de contenidos vs televisión abierta y de paga. Ift.Org.Mx. http://www.ift.org.mx/ sites/default/files/ott_pdf_0.pdf

» Fandom.com. (s. f.). Stranger Things Wiki | Fandom. Recuperado https://strangerthings.fandom.com/es/wiki/Stranger_Things Wiki

» Flores-Heymann, B. (2016, 26 septiembre). Síndrome de transmedialidad múltiple. Linkedln Pulse. https://www.linkedin.com/pulse/s\%C3\%ADndrome-de-transmedialidad-m\%C3\%BAltiple-bernardo-flores-heymann/

» Forbes México / Notimex. (2019, 19 abril). La televisión en México ya se consume diferente. Forbes.com.mx. https://www.forbes. com.mx/la-television-en-mexico-ya-se-consume-diferente

»IFT. (2019, September 17). SEIS DE CADA 10 USUARIOS PASAN HASTA DOS HORAS DIARIAS VIENDO CONTENIDO EN PLATA- 
FORMAS DE VIDEO POR INTERNET. Ift.Org.Mx. http://www.ift. org.mx/comunicacion-y-medios/comunicados-ift/es/seis-de-cada10-usuarios-pasan-hasta-dos-horas-diarias-viendo-contenido-enplataformas-de-video-por

» Jenkins, H. (2008). Convergence Culture. Amsterdam University Press.

» Jenkins, H. (2010, 21 junio). Transmedia Education: the 7 Principles Revisited. Henry Jenkins. http://henryjenkins.org/ blog/2010/06/transmedia_education_the_7_pri.html

» Jiménez, R. (2019, 10 julio). Conoce los cuartos de Stranger Things en la Ciudad de México. El Universal. https://www.eluniversal.com. $\mathrm{mx} /$ destinos/conoce-los-cuartos-de-stranger-thingsen-la-ciudad-de-mexico

» Lobato, Ramon (2019). Netflix Nations. The Geography of Digital Distribution (en inglés). Nueva York: New York University Press.

» Merca2.0. https://www.merca20.com/error-netflix-promocionar-stranger-things/

» Nava, I. (2019, 5 julio). El error de Netflix al intentar promocionar a Stranger Things. Revista

» Negroponte,N. (1995). El mundo digital. Ediciones B (ed.orig.:Being Digital, NuevaYork,Vintage Books, 1995).

» Netflix. (2019, 8 julio). Captura de pantalla [Fotografía]. Twitter com. https://twitter.com/netflix/status/1148359444188712960

» Netflix. (2019b, julio 15). Jaime Maussan y Cositas vs el Mind Flayer | Stranger Things | Netflix [Vídeo]. YouTube. https://www.youtube.com/watch?v=xQ77sza2XK0

» Nuñez, A. (2019, 12 julio). Netflix lanza un podcast sobre Stranger Things. Movie Network. https://movienetworkpr.com/netflix-lanza-un-podcast-sobre-stranger-things/

» Pérez, E. (2017, 4 octubre). Stranger Things: The Game, un RPG retro y ochentero a la altura de la serie. Xataka Android. https:// www.xatakandroid.com/juegos-android/stranger-things-the-game-un-rpg-retro-y-ochentero-a-la-altura-de-la-serie

» Radio, W. (2019, 25 julio). La radio extraña: Una adaptación de Stranger Things para W Radio. W Radio México. https://wradio.com.mx/radio/2019/07/02/entretenimiento/1562090676 225048.htm

» Ramírez, G. (2020, 25 enero). Netflix alcanza a 6 millones en México. El Sol de México. https://www.elsoldemexico.com.mx/finanzas/netflix-alcanza-a-6-millones-en-mexico-4747641.html

» Sacristán, L. (2019, 30 agosto). «Stranger Things 3: The Game», el nuevo juego basado en la serie de Netflix, ya está disponible en iOS y...Xataka Móvil. https://www.xatakamovil.com/aplicaciones/ stranger-things-3-the-game-nuevo-juego-basado-serie-netflix-esta-disponible-ios-android

» Scolari, C. (2013). Hipermediaciones: elementos para una teoría de la comunicación digital interactiva.Editorial Gedisa.

» Shapiro, A. (2020, April 22). Netflix Adds 15.8 Million Subscribers In First Quarter. Forbes. https://www.forbes.com/sites/arielshapiro/2020/04/21/netflix-stock-up-5-after-hours-reports-158-miIlion-additional-subscribers/

" Smith, Michael D. y Telang, Rahul (2016). "1: House of Cards». Streaming, Sharing, Stealing. Big Data and the Future of
Entertainment (en inglés). MIT Press. ISBN 9780262034791.

» Stelter, B. (2013, 19 julio). Netflix Does Well in 2013 Primetime Emmy Nominations. The New York Times. https://artsbeat.blogs. nytimes.com/2013/07/18/watching-for-the-2013-primetime-emmy-nominations/

» Unocero. (2019, 14 mayo). Netflix estrena podcast español para los que "no tienen nada que ver" en la plataforma. https://www. unocero.com/software/nada-que-ver-podcast-netflix/

» Yengin, Deniz (Octubre de 2016). «TRANSFORMATION OF LEISURE TIME IN NEW MEDIA: BINGE WATCH». The Turkish Online Journal of Design, Art and Communication - TOJDAC. 